\title{
Method of Perturbative-PIC Simulation for Interactions between a Particle Bunch and Its Coherent Synchrotron Radiation *
}

\author{
J. Shi, Dept. of Physics \& Astronomy, The University of Kansas, Lawrence, KS 66045, USA \\ G.H. Hoffstetter, Dept. of Physics, Cornell University, Ithaca, NY 14850, USA
}

\begin{abstract}
A self-consistent simulation method is developed for the study of coherent synchrotron radiation effects by using a perturbation expansion of retarded radiation field and the particle-in-cell method. With this method, the particleradiation interaction can be calculated self-consistently without memorizing the history of the bunch particle distribution during a beam tracking.
\end{abstract}

\section{INTRODUCTION}

In the study of collective particle-field interactions, the chief of difficulties is that the calculation of the field requires a knowledge of particle distribution while the distribution itself is perturbed by the field. Currently, the most effective numerical method for a self-consistent treatment of such a collective interaction is the particle-in-cell (PIC) method in which the time evolution of the particle distribution is calculated on a mesh in configuration space by using a large number of macroparticles [1]. For the interaction between an ultra short bunch and its coherent synchrotron radiation (CSR), however, one additional difficulty for a self-consistent treatment is the retardation of the radiation field as the particle-radiation interaction involves a retarded bunch particle distribution. A direct use of the PIC method is very inefficient, if not impossible, because with a reasonable density of the mesh and a reasonable number of macroparticles, keeping the history of the particle distribution in computer memories during a beam tracking is technically impractical (expect maybe in the case of a very short beam path.)

To overcome the difficulty of the retardation in a selfconsistent treatment of the CSR problem, we have developed a perturbative-PIC method based on a perturbation expansion of the retarded radiation field. For the perturbation expansion of the radiation field, we utilize the fact that the time dependence of a bunch particle distribution has two significantly different time scales. A fast time scale of the distribution is related to the linear dynamics of a bunch as its centroid moves along the lattice and its beam sizes vary with lattice functions while a slow time scale of the distribution is of the slow beam-size growth due to nonlinear perturbations. The retardation on the fast time scale can be eliminated from the distribution analytically since the linear dynamics is known. As the retardation of the radiation is usually much shorter than the slow time scale of the distribution, the retardation on the slow time scale in the

\footnotetext{
* Work supported by the US Department of Energy under Grant No. DE-FG02-04ER41288.
}

distribution can be approximated by an expansion in terms of the retardation. The use of the PIC method provides a smooth distribution constructed on a mesh in configuration space so that the expansion of the retarded radiation field can be calculated numerically on the mesh with a desired accuracy. With this method, the retarded radiation field can be calculated self-consistently without a need of memorizing the history of the distribution during a beam tracking.

In a simulation of the CSR problem, the radiation field can be calculated either directly in the form of the Lorentz force or indirectly through the Liénard-Wiechert potential in configuration or frequency space. The direct force calculation is faster but it is more prone to the numerical singularity of the radiation field as the radiation from an ultrarelativistic charge is concentrated inside a radiation cone with an angular width of $\sim \gamma^{-1}$. This perturbative-PIC method can be used for both the direct and indirect calculation of the radiation field. Note that the use of the PIC method can significantly reduce the numerical singularity of the radiation field.

\section{PARTICLE-RADIATION INTERACTION}

Consider a bunch that moves along a plane circular design orbit of radius $R$ with a constant speed of $v_{0}$, where $\beta_{0}=v_{0} / c \sim 1$. Let $\vec{X}(t)=\vec{X}_{c}(s)+\vec{x}(s)$ be the global coordinate of a bunch particle in the lab frame, where $s=v_{0} t$ is the independent ("time") variable, $\vec{X}_{c}(s)$ is the global coordinate of the bunch centroid along the design orbit, and $\vec{x}=(x, y, z)$ is the coordinate of the particle respect to the bunch centroid where $x, y$, and $z$ are the horizontal, vertical, and longitudinal coordinates, respectively. Let $\tilde{f}(\vec{X}, \vec{P}, s)$ be the bunch particle distribution in the phase space of the global coordinate. The Lorentz force on a test particle at $\vec{X}$ due to the synchrotron radiation from a bunch or the vector potential of the radiation field at $\vec{X}$ can be calculated from

$$
\vec{F}_{\mathrm{SR}}=\int_{-\infty}^{\infty} \vec{G}\left(\vec{X}-\vec{X}_{1}, s, \tau_{s}\right) \tilde{f}\left(\vec{X}_{1}, \vec{P}_{1}, s-\tau_{s}\right) d \vec{X}_{1} d \vec{P}_{1}
$$

where $\vec{G}\left(\vec{X}-\vec{X}_{1}, s, \tau_{s}\right)$ is the Lorentz force on a test charge at $(\vec{X}, s)$ (for the direct calculation of the force) or the Liénard-Wiechert potential (for the calculation of the potential) of the electromagnetic field radiated from a charge at $\left(\vec{X}_{1}, s_{1}\right)$ in the lab frame, and $\tau_{s}=s-s_{1}=\beta_{0}\left|\vec{X}-\vec{X}_{1}\right|$ is the retardation. Note that $\vec{G}$, in general, could also be a function of $\vec{P}_{1}$ of the particle that radiates the field at $s_{1}$ and of $\vec{P}$ of the test particle at $s$. For an ultra-relativistic beam, the dependence of the velocities or accelerations in $\vec{G}$ can 
be well approximated by the velocity or acceleration of the bunch centroid motion and, therefore, $\vec{G}$ does not depend on $\vec{P}_{1}$ or $\vec{P}$ in Eq. (1). The integral in Eq. (1) can thus be simplified as an integral with the bunch particle density in configuration space.

\section{a. Elimination of Fast Time Dependence of $\tilde{f}(\vec{X}, \vec{P}, s)$}

The fast time scale in $\tilde{f}(\vec{X}, \vec{P}, s)$ is of the linear beam dynamics that includes the motion of the bunch centroid and the linear variation of the beam size around an accelerator. Since the linear dynamics is known, the retardation of the fast time scale in $\tilde{f}\left(\vec{X}_{1}, \vec{P}_{1}, s-\tau_{s}\right)$ can be eliminated by transferring to the normalized variables. Let $\vec{x}=\left(\sqrt{\beta_{x}(s)} q_{1}, \sqrt{\beta_{y}(s)} q_{2}, q_{3}\right)$ and $\vec{x}_{1}=$ $\left(\sqrt{\beta_{x}\left(s-\tau_{s}\right)} q_{1}^{\prime}, \sqrt{\beta_{y}\left(s-\tau_{s}\right)} q_{2}^{\prime}, q_{3}^{\prime}\right)$, where $\beta_{x}(s)$ and $\beta_{y}(s)$ are beta functions with known $s$-dependence. Since the transformation from $\left(\vec{X}_{1}, \vec{P}_{1}\right)$ to $\left(\vec{q}^{\prime}, \vec{p}^{\prime}\right)$ is symplectic, where $\vec{p}^{\prime}$ is the momentum conjugate to $\vec{q}^{\prime}$, the integral in Eq. (1) simply becomes

$$
\vec{F}_{\mathrm{SR}}(\vec{q}, s)=\int_{-\infty}^{\infty} \vec{G}_{1}\left(\vec{q}, \vec{q}^{\prime}, \tau_{s}\right) \rho\left(\vec{q}^{\prime}, s-\tau_{s}\right) d \vec{q}^{\prime}
$$

where $\vec{G}_{1}\left(\vec{q}, \vec{q}^{\prime}, \tau_{s}\right)=\vec{G}\left(\vec{X}-\vec{X}_{1}, s, \tau_{s}\right)$,

$$
\rho\left(\vec{q}^{\prime}, s-\tau_{s}\right)=\int_{-\infty}^{\infty} f\left(\vec{q}^{\prime}, \vec{p}^{\prime}, s-\tau_{s}\right) d \vec{p}^{\prime},
$$

and $f(\vec{q}, \vec{p}, s)$ is the bunch particle distribution in the normalized phase space. Note that $\tau_{s}$ is a function of $\left(\vec{q}, \vec{q}^{\prime}\right)$ and does not depend on $\vec{p}^{\prime}$. Without any nonlinearity and if the bunch matches with the linear lattice initially, $\partial \rho(\vec{q}, s) / \partial s=0$. With nonlinear perturbations such as CSR, $\rho(\vec{q}, s)$ may weakly depend on $s$ explicitly and the retardation of the weak explicit $s$-dependence of $\rho\left(\vec{q}, s-\tau_{s}\right)$ can be treated perturbatively. If a bunch is mismatched with the linear lattice, as long as the oscillation of the bunch due to the mismatch is not significant during the retardation, the perturbative treatment of the retardation for $\rho\left(\vec{q}, s-\tau_{s}\right)$ is still valid.

\section{b. Expansion of Explicit $\tau_{s}$-Dependence of $\rho\left(\vec{q}, s-\tau_{s}\right)$}

Consider the situation that the emittance growth is small in the time scale of the retardation, i.e. $\left\langle\left(\tau_{\max } / \rho\right)(\partial \rho / \partial s)\right\rangle \sim\left(\tau_{\max } / \sigma\right)(d \sigma / d s)<<1$, where $\tau_{\max }=\max \left(\tau_{s}\right)$ and $\sigma$ is the r.m.s. beam emittance. The retardation of the explicit $s$-dependence of $\rho\left(\vec{q}, s-\tau_{s}\right)$ can be expanded as

$$
\rho\left(\vec{q}, s-\tau_{s}\right)=\sum_{n=0} \frac{1}{n !} \frac{\partial^{n} \rho(\vec{q}, s)}{\partial s^{n}}\left(-\tau_{s}\right)^{n}
$$

Note that this expansion preserves the normalization condition of $\rho$. The integral in Eq. (2) can then be calculated through the expansion as

$$
\vec{F}_{\mathrm{SR}}(\vec{q}, s)=\sum_{n=0} \vec{F}_{n}(\vec{q}, s)
$$

where

$$
\vec{F}_{n}=\frac{1}{n !} \int_{-\infty}^{\infty} \vec{G}_{1}\left(\vec{q}, \vec{q}^{\prime}, \tau_{s}\right)\left(-\tau_{s}\right)^{n} \frac{\partial^{n} \rho\left(\vec{q}^{\prime}, s\right)}{\partial s^{n}} d \vec{q}^{\prime}
$$

Note that the convergence of the expansion in Eq. (4) is usually better than the expansion of the distribution in Eq. (3) since the integration in Eq. (5) smoothes the time evolution of a numerically constructed distribution in a PIC simulation. If the time step in the simulation is small enough, keeping the first-order term is sufficient, then

$$
\vec{F}_{\mathrm{SR}}(\vec{q}, s) \simeq \vec{F}_{0}(\vec{q}, s)+\vec{F}_{1}(\vec{q}, s)+O\left(\frac{\tau_{\max }^{2}}{\sigma} \frac{\partial^{2} \sigma}{\partial s^{2}}\right)
$$

where $\vec{F}_{0}$ can be evaluated with the standard PIC method. To evaluate $\vec{F}_{1}$, one needs $\partial \rho / \partial s$ that could be obtained directly on the mesh with a numerical differentiation during a PIC simulation. The direct calculation of $\partial \rho / \partial s$, however, requires a memorization of $\rho$ for a couple of time steps in the simulation and could be numerically unstable at some mesh points if the time evolution of the numerically constructed distribution is not smooth enough. In fact, it is not really necessary to calculate $\partial \rho / \partial s$ directly. Because $\vec{G}_{1}\left(\vec{q}, \vec{q}^{\prime}, \tau_{s}\right)$ does not depends on the momenta, $\partial \rho / \partial s$ in Eq. (5) can be converted into the derivatives of the unperturbed Hamiltonian (Hamiltonian of the linear motion) with respect to phase-space variables and can thus be obtained in a closed form. For this purpose, we start with the Liouville's equation for the bunch particle distribution,

$$
\frac{\partial f}{\partial s}=-\frac{d \vec{p}}{d s} \cdot \frac{\partial f}{\partial \vec{p}}-\frac{d \vec{q}}{d s} \cdot \frac{\partial f}{\partial \vec{q}}
$$

In beam tracking, the Lorenz force from the synchrotron radiation can be approximated as momentum kicks applied on beam particles successively along the beam path. With this kick approximation, the equation of motion is

$$
\begin{aligned}
\frac{d \vec{q}}{d s} & =\frac{\partial H_{0}}{\partial \vec{p}} \\
\frac{d \vec{p}}{d s} & =-\frac{\partial H_{0}}{\partial \vec{q}}+\sum_{k} \vec{K}_{\mathrm{SR}}(\vec{q}, s) \delta\left(s-s_{k}\right)
\end{aligned}
$$

where $H_{0}$ is the Hamiltonian for the motion without the radiation field, $\vec{K}_{\mathrm{SR}}$ is the kick of the radiation field $\vec{F}_{\mathrm{SR}}$, and $s_{k}$ is the location of the radiation kick on the beam path. With an ultra-relativistic beam, $\vec{F}_{\mathrm{SR}}$ does not depend on $\vec{p}$ explicitly and the symplectic condition is preserved in Eq. (8). Substituting Eq. (8) into Eq. (7) and integrating over momenta on the both sides of Eq. (7) yields

$$
\frac{\partial \rho}{\partial s}=-\frac{\partial}{\partial \vec{q}} \cdot\left\langle\frac{\partial H_{0}}{\partial \vec{p}}\right\rangle_{\vec{p}}
$$

where $\langle\cdots\rangle_{\vec{p}}$ is the average over the beam in the momentum space. Since the Hamiltonians for other nonlinear perturbations does not depend on the momenta neither, only the Hamiltonian of the linear motion is relevant 
to $\partial H_{0} / \partial \vec{p}$ and $\partial \rho / \partial s$ does not depend on nonlinear perturbations explicitly. With the unperturbed Hamiltonian of a linear lattice for an ultra-relativistic beam, one has $\left\langle\partial H_{0} / \partial p_{1}\right\rangle_{\vec{p}}=\left\langle p_{1}\right\rangle_{\vec{p}} / \beta_{x},\left\langle\partial H_{0} / \partial p_{2}\right\rangle_{\vec{p}}=\left\langle p_{2}\right\rangle_{\vec{p}} / \beta_{y}$, and $\left\langle\partial H_{0} / \partial p_{3}\right\rangle_{\vec{p}}=-\left[1+\beta_{x}^{1 / 2} q_{1} / R\right] \rho$. Note that $\langle\vec{p}\rangle_{\vec{p}}=$ 0 if a bunch is symmetric in $\vec{p}$. With nonlinear perturbations, a bunch usually cannot maintain such a mirror symmetry in each dimension of the momentum space and, in general, $\langle\vec{p}\rangle_{\vec{p}} \neq 0$ even in the case that the bunch is initially symmetric. In the PIC simulation, $\langle\vec{p}\rangle_{\vec{p}}$ as functions of $\vec{q}$ can be constructed on the mesh using a similar computational procedure as that for $\rho(\vec{q}, s)$. Substituting Eq. (9) into $\vec{F}_{1}$ in Eq. (5) and using integration by parts yields

$$
\vec{F}_{1}(\vec{q}, s)=-\sum_{i=1}^{3} \int_{-\infty}^{\infty} \overrightarrow{\mathcal{G}}_{i}\left(\vec{q}, \vec{q}^{\prime}, \tau_{s}\right)\left\langle\frac{\partial H_{0}}{\partial p_{i}}\right\rangle_{\vec{p}} d \vec{q}^{\prime}
$$

where

$$
\overrightarrow{\mathcal{G}}_{i}\left(\vec{q}, \vec{q}^{\prime}, \tau_{s}\right)=\left(\vec{G}_{1}+\frac{\partial \vec{G}_{1}}{\partial \tau_{s}}\right) \frac{\partial \tau_{s}}{\partial q_{i}^{\prime}}+\tau_{s} \frac{\partial \vec{G}_{1}}{\partial q_{i}^{\prime}}
$$

Note that $\vec{G}_{1}$ is a function of $\left(\vec{q}, \vec{q}^{\prime}, \tau_{s}\right)$ and $\tau_{s}$ is a function of $\left(\vec{q}, \vec{q}^{\prime}\right)$. The $\vec{q}^{\prime}$-dependence of $\tau_{s}$ can be solved from

$$
\tau_{s}=\beta_{0}\left|\vec{X}_{c}(s)-\vec{X}_{c}\left(s-\tau_{s}\right)+\vec{x}-\vec{x}_{1}\right|
$$

either numerically during the tracking or approximately in a closed form. The closed form of $\partial \tau_{s} / \partial \vec{q}^{\prime}$ can be obtained by taking the derivative of both sides of Eq. (11) with respect to $\vec{q}^{\prime}$. As the closed forms of $\vec{G}_{1}$ and $\overrightarrow{\mathcal{G}}_{i}$ are available, the quadratures for $\vec{F}_{0}$ in Eq. (5) and $\vec{F}_{1}$ in Eq. (10) can be evaluated after $\rho(\vec{q}, s)$ and $\langle\vec{p}\rangle_{\vec{p}}$ are constructed on the mesh during a PIC simulation.

\section{PIC METHOD FOR EVALUATING $\overrightarrow{\boldsymbol{F}}_{\boldsymbol{S R}}$}

In simulation, a particle bunch is represented by a large number of macroparticles in phase space. The initial particle distribution can be any distribution but is usually a Gaussian. During the tracking, the synchrotron radiation field is calculated with a numerically constructed self-consistent bunch particle distribution by using the PIC method. In the traditional PIC simulation, the particle density $\rho(\vec{q}, s)=\langle 1\rangle_{\vec{p}}$ is constructed on a mesh in configuration space with the macroparticles by using a cloud-in-cell technique [1]. Similarly, $\langle\vec{p}\rangle_{\vec{p}}$ as a function of $\vec{q}$ is also constructed on the mesh for the evaluation of $\vec{F}_{1}$. For example, in the case of a three-dimensional mesh that covers the transverse and longitudinal configuration space, both $\rho(\vec{q}, s)$ and $\langle\vec{p}\rangle_{\vec{p}}$ on the mesh can be obtained by assigning each macroparticle to its eight nearest mesh points based on the weights of the macroparticle at the mesh points. The contributions of a macroparticle to $\rho(\vec{q}, s)$ or $\langle\vec{p}\rangle_{\vec{p}}$ at a mesh point are simply the weight of the macroparticle at that point or the weight multiplied by the momentum of the macroparticle, respectively. After $\rho(\vec{q}, s)$ and $\langle\vec{p}\rangle_{\vec{p}}$ at all mesh points are known, $\vec{F}_{0}$ and $\vec{F}_{1}$ are first calculated at all mesh points with numerical integrations of Eq. (5) with $n=0$ and Eq. (10). The radiation field is then interpolated to positions of every macroparticles for beam tracking. One advantage of the PIC calculation with a direct integration of the field is the possibility to use a relatively small mesh. Since usually almost all beam particles are inside a phasespace region of three to four r.m.s. beam sizes, a mesh that extends to six r.m.s. beam sizes and is dynamical adapted to beam-size growth is usually larger enough for the PIC simulation.

\section{FINAL REMARKS}

Because of the difficulty in treating the retardation numerically, most simulation codes currently developed for the CSR problem have adapted following approaches [2]: (a) non-self-consistent, (b) equivalently keeping only the 0th-order term $\vec{F}_{0}$ in Eq. (4) for the radiation field, (c) direct tracking of a limited number of macroparticles and the particle-radiation interaction is calculated with particle-toparticle individually, and (d) solving the Vlasov-Maxwell equation in phase space. For the approach (b), it is not clear how important the retardation of the high-order variation of the distribution due to CSR. For the approach (c), the calculation of a collective interaction with particle-toparticle individually has been known to be not reliable due to a nonsmooth field. For the approach (d), the challenge is how to solve the Vlasov-Maxwell equation with a reasonable efficiency for the case of a long beam path such as in a storage ring. The perturbative-PIC method presented here provides an order-by-order approach to examine the importance of the retardation to the collective CSR effects, especially the instabilities induced by CSR. It should be noted that our current approach of the perturbative-PIC method neglects the effect of beam pipe on the radiation field and, therefore, can only be applied to the unshielded CSR problem.

To check our perturbatice-PIC code, we have calculate the radiation field of a one-dimensional Gaussian bunch (a line charge) and the result is consistent with the analytic solution [3]. Due to a limited space here, the numerical result will be discussed elsewhere.

\section{ACKNOWLEDGMENTS}

J. Shi is thankful to R. Li for many stimulating discussions and comments.

\section{REFERENCES}

[1] R.W. Hockney and J.W. Eastwood, Computer Simulation Using Particles, (McGraw-Hill, New York, 1981).

[2] G. Bassi, "Overview of CSR codes", Nucl. Instr. \& Meth. A577, 189 (2006), and references herein.

[3] Ya.S. Derbenev and V.D. Shiltsev, SLAC Report No. SLACPUC-7181, (1996). 